\title{
Analytical Approximation for Newtonian Boson Stars in Four and Five Dimensions
}

-A Poor Person's Approach to Rotating Boson Stars-

\author{
Nahomi Kan* \\ National Institute of Technology, Gifu College, Motosu-shi, Gifu 501-0495, Japan \\ Kiyoshi Shiraishi ${ }^{\dagger}$ \\ Graduate School of Sciences and Technology for Innovation, \\ Yamaguchi University, Yamaguchi-shi, Yamaguchi 753-8512, Japan
}

(Dated: July 9, 2018)

\begin{abstract}
In this paper, we study rotating boson stars in the large coupling limit as well as in the Newtonian limit. We investigate the equilibrium solutions in four and five dimensions by adopting some analytical approximations. We show that the relations among the radius, angular momentum, Newtonian energy, and quadrupole moment (for the four-dimensional solution) of the boson star can be qualitatively realized for the minimal number of boson-star parameters.
\end{abstract}

PACS numbers: 04.25.-g, 04.40.-b, 04.50.-h, 04.50.Cd, 05.30.Jp, 11.10.-z, 11.10.Kk, 11.25.Mj, 11.27.+d .

\footnotetext{
* kan@gifu-nct.ac.jp

† shiraish@yamaguchi-u.ac.jp
} 


\section{INTRODUCTION}

Along with the development of observational cosmology, including the recent gravitywave detection, the theoretical study of compact objects is actively advancing apace. Thus far, remarkable progress has been made in the study of universal relations between physical quantities of compact objects, such as the angular momentum, quadrupole moment, mass, and radius.

The study of self-gravitating systems is very interesting because the characteristics of general relativity or the modified gravity appear in the physical quantities of the system. For example, Brihaye, Hartmann, and collaborators argued about a boson star solution in a higher-dimensional spacetime [1-3].

From the perspective of particle cosmology, the boson star [4-6] is one of the candidates for dark matter [7-11]. The (1+3)-dimensional boson star was studied as the simplest model of a self-gravitating system and the Newtonian treatment of gravitating bosons has been often discussed [4]. Although the difference between the Newtonian treatment and the general relativistic model is significant, the Newtonian treatment is known to enable the evaluation of the mass of the boson star qualitatively. It is necessary to examine the Newtonian approximation in higher-dimensional boson systems, since a substantial understanding is important to check whether a universal property of compact objects exists even in the modified gravity theory.

We discuss the qualitative behavior of models such as a four-dimensional rotating boson star (which has been studied in the general relativistic model [12-16]), a boson star in dimensions with compactified space and five-dimensional boson stars using Newtonian approximation. We study the system of a scalar field with large self-interaction [17] in this paper. A deep understanding of the basic aspects of self-gravitating systems, which is independent of a possible correction in the gravity theory, is expected from this study.

The present paper is organized as follows. In Sec. II, we obtain the action, the Hamiltonian, and field equations for a model of a self-interacting, gravitating boson field in the Newtonian limit. The large coupling limit of the model is defined in Sec. III and we obtain the spherical solution for a boson star and discuss its mass. In Sec. IV, approximated solutions for rotating boson stars are obtained, and the relation among their physical quantities is studied. The boson star in the Kaluza-Klein background is considered in Sec. V, and the 
stability against a small variation along with an extra dimension is discussed. In Sec. VI, five-dimensional boson stars in the Newtonian limit are investigated. The last section is devoted to a summary and future prospects.

\section{THE NEWTONIAN LIMIT}

We consider a system of self-interacting, gravitating scalar bosons of mass $m$ governed by the following field-theoretical action;

$$
S=\int d^{4} x \sqrt{-g} \mathcal{L}=\int d^{4} x \sqrt{-g}\left[\frac{1}{16 \pi G} R-|\partial \phi|^{2}-m^{2}|\phi|^{2}-\frac{1}{2} \tilde{\lambda}|\phi|^{4}\right],
$$

where $d^{4} x=d t d^{3} \boldsymbol{x}, G$ is the Newton constant, $R$ is the Ricci scalar, $\tilde{\lambda}$ is the dimensionless scalar self-coupling constant, and $|\partial \phi|^{2} \equiv g^{\mu \nu}\left(\partial_{\mu} \phi\right)^{*}\left(\partial_{\nu} \phi\right)$.

By the variational principle, we derive the Einstein equation from the action as

$$
R_{\nu}^{\mu}-\frac{1}{2} \delta_{\nu}^{\mu} R=8 \pi G T_{\nu}^{\mu}
$$

where the energy-momentum tensor in the system is given by

$$
T_{\mu \nu}=\left[\partial_{\mu} \phi^{*} \partial_{\nu} \phi+\partial_{\nu} \phi^{*} \partial_{\mu} \phi-g_{\mu \nu}|\partial \phi|^{2}-g_{\mu \nu} m^{2}|\phi|^{2}\right]-g_{\mu \nu} \frac{1}{2} \tilde{\lambda}|\phi|^{4} .
$$

The equation of motion for the complex scalar field $\phi$ is given by

$$
\square \phi-m^{2} \phi-\tilde{\lambda}|\phi|^{2} \phi=0,
$$

where $\square \phi \equiv \frac{1}{\sqrt{-g}} \partial_{\mu}\left(\sqrt{-g} g^{\mu \nu} \partial_{\nu} \phi\right)$ is the covariant d'Alembertian.

The Newtonian limit can be attained by assuming that the spacetime metric in the weak field approximation can be written as

$$
g_{00} \approx-(1+2 \Phi), \quad \sqrt{-g} \approx 1
$$

where $\Phi$ is the Newtonian gravitational potential.

Assuming further that a complex scalar field has a nearly harmonic time dependence expressed by ${ }^{1}$

$$
\phi=\frac{1}{\sqrt{2 m}} \psi(\boldsymbol{r}, t) e^{-i m t},
$$

\footnotetext{
1 Since we consider the Newtonian limit, the frequency $\omega$ is close to the scalar field mass $m$.
} 
we obtain the (non-linear) Schrödinger equation

$$
i \dot{\psi}=-\frac{1}{2 m} \nabla^{2} \psi+m \Phi \psi+\frac{\tilde{\lambda}}{4 m^{2}}|\psi|^{2} \psi
$$

as the Newtonian limit of Eq. (2.4), where $\nabla^{2}$ is the Laplacian in the flat space and the dot indicates the time derivative. In the present limit, the Einstein equations reduce to the Poisson equation

$$
\nabla^{2} \Phi=4 \pi G m|\psi|^{2}
$$

The Newtonian treatment of the Lagrangian and Hamiltonian is as follows. We find the following Newtonian action in the limit:

$$
S \cong \int d t d^{3} \boldsymbol{r}\left[-\frac{1}{8 \pi G}(\nabla \Phi)^{2}+i \psi^{*} \dot{\psi}-\frac{1}{2 m}|\nabla \psi|^{2}-m \Phi|\psi|^{2}-\frac{1}{8} \frac{\tilde{\lambda}}{m^{2}}|\psi|^{4}\right]
$$

where $(\nabla \Phi)^{2} \equiv \nabla \Phi \cdot \nabla \Phi$ and the symbol $\cong$ indicates that some surface terms have been omitted. Therefore, the Hamiltonian of the system is derived as

$$
\hat{H}(\psi, \Phi)=\int d^{3} \boldsymbol{r} \mathcal{H}=\int d^{3} \boldsymbol{r}\left[\frac{1}{8 \pi G}(\nabla \Phi)^{2}+\frac{1}{2 m}|\nabla \psi|^{2}+m \Phi|\psi|^{2}+\frac{1}{8} \frac{\tilde{\lambda}}{m^{2}}|\psi|^{4}\right] .
$$

On the other hand, the Newtonian number of particles is expressed as

$$
\hat{N} \equiv \int d^{3} \boldsymbol{r}|\psi|^{2}
$$

In addition, we require the condition $\hat{N}=N$, i.e., the condition that the system contains $N$ scalar bosons. Then, we consider $\delta\{\hat{H}-\mu(\hat{N}-N)\}=0$ as an equation for the scalar matter field in the mean field approximation, where $\mu$ is a Lagrange multiplier.

Now, we obtain two coupled equations for the stationary gravitational field and the matter field as follows:

$$
\begin{aligned}
& \nabla^{2} \Phi=4 \pi G m|\psi|^{2}, \\
& -\frac{1}{2 m} \nabla^{2} \psi+m \Phi \psi+\frac{\tilde{\lambda}}{4 m^{2}}|\psi|^{2} \psi=\mu \psi .
\end{aligned}
$$

Therefore, the system is reduced in the Newtonian limit to the (non-linear) SchrödingerPoisson system.

In the subsequent sections of this paper, we will concentrate on the large coupling limit to extract analytic results for compact objects. 


\section{LARGE COUPLING LIMIT AND THE SPHERICAL SOLUTION}

Here, we consider the large coupling limit [17]. We assume the case of $\Lambda \gg 1$, where

$$
\Lambda=\frac{\tilde{\lambda}}{8 \pi G m^{2}} .
$$

In addition, if we introduce the following quantities

$$
\boldsymbol{r}_{*}=\frac{m}{\sqrt{\Lambda}} \boldsymbol{r}, \quad \Psi=\sqrt{\frac{4 \pi G \Lambda}{m}} \psi, \quad \mu_{*}=\frac{\mu}{m},
$$

the set of equations reduces to the simple form

$$
\begin{aligned}
& \nabla_{*}^{2} \Phi=|\Psi|^{2}, \\
& -\frac{1}{2 \Lambda} \nabla_{*}^{2} \Psi+\Phi \Psi+\frac{1}{2}|\Psi|^{2} \Psi=\mu_{*} \Psi,
\end{aligned}
$$

where $\nabla_{*}^{2}$ is the rescaled Laplacian expressed in terms of the coordinate $\boldsymbol{r}_{*}$.

In the limit of $\Lambda \rightarrow \infty$, equation (3.4) further reduces to

$$
\Phi \Psi+\frac{1}{2}|\Psi|^{2} \Psi=\mu_{*} \Psi .
$$

We can interpret the solutions of (3.5) as follows. In the region outside the boson star, the solution is $\Psi \equiv 0$. In the region inside the boson star, i.e., in the region of $|\Psi|>0$, the solution is expressed by

$$
\Phi=\mu_{*}-\frac{1}{2} \rho,
$$

where the normalized density function is defined as

$$
\rho \equiv|\Psi|^{2} .
$$

Note that $\mu_{*}$ indicates the value of the gravitational potential at the surface of the boson star, where $\rho=0$. If the relation (3.6) is substituted in the Poisson equation (3.3), we obtain the linear differential equation

$$
\nabla_{*}^{2} \rho+2 \rho=0,
$$

which is valid in the region inside the boson star, while $\rho=0$ outside the star. ${ }^{2}$ Because of Eq. (2.11), the solution of the linear equation (3.8) should be normalized as

$$
N=\frac{\sqrt{\Lambda}}{4 \pi G m^{2}} \int d^{3} \boldsymbol{r}_{*} \rho\left(\boldsymbol{r}_{*}\right) .
$$

\footnotetext{
${ }^{2}$ Note that, in the large coupling limit, the boson star has no asymptotic tail outside the star [15, 17].
} 
Now, we consider the spherically symmetric solution of the system. Then, the equation (3.8) can be rewritten as

$$
\frac{1}{r_{*}} \frac{d^{2}}{d r_{*}^{2}}\left(r_{*} \rho\right)+2 \rho=0
$$

where $r_{*}=\sqrt{\boldsymbol{r}_{*} \cdot \boldsymbol{r}_{*}}$. The normalized solution for $\rho\left(r_{*}\right)$ is analytically expressed as

$$
\rho\left(r_{*}\right)=\left\{\begin{array}{cc}
\frac{4 \pi G m^{2}}{\sqrt{\Lambda}} \frac{N}{\sqrt{2} \pi^{2}} \frac{\sin \sqrt{2} r_{*}}{\sqrt{2} r_{*}} & \left(r_{*}<\pi / \sqrt{2}\right) \\
0 & \left(r_{*}>\pi / \sqrt{2}\right)
\end{array} .\right.
$$

The solution for the gravitational potential $\Phi\left(r_{*}\right)$ can be found by Eq. (3.6) for $r_{*} \leq \pi / \sqrt{2}$. We should choose the value of $\mu_{*}$ so that $\Phi\left(r_{*}\right)$ matches the Newton potential at $r_{*}=\pi / \sqrt{2}$. Hence, we find

$$
\Phi\left(r_{*}\right)=\left\{\begin{array}{cc}
-\frac{\sqrt{2}}{\pi} \frac{G m^{2} N}{\sqrt{\Lambda}}-\frac{2 \pi G m^{2}}{\sqrt{\Lambda}} \frac{N}{\sqrt{2} \pi^{2}} \frac{\sin \sqrt{2} r_{*}}{\sqrt{2} r_{*}} & \left(r_{*}<\frac{\pi}{\sqrt{2}}\right) \\
-\frac{G m^{2} N}{\sqrt{\Lambda} r_{*}} & \left(r_{*}>\frac{\pi}{\sqrt{2}}\right)
\end{array} .\right.
$$

Note that $\mu_{*}$ takes a negative value in general.

The Newtonian energy $E$ of the system in the large coupling limit can be expressed from Eq. (2.10) as

$$
E=\frac{\sqrt{\Lambda}}{4 \pi G m} \int d^{3} \boldsymbol{r}_{*}\left[\frac{1}{2}\left(\nabla_{*} \Phi\right)^{2}+\Phi|\Psi|^{2}+\frac{1}{4}|\Psi|^{4}\right] .
$$

Substituting the solution of Eqs. (3.8) and (3.6) into this equation, we obtain

$$
E \cong \frac{\sqrt{\Lambda}}{4 \pi G m} \int d^{3} \boldsymbol{r}_{*}\left[\frac{1}{2} \Phi \rho+\frac{1}{4} \rho^{2}\right]=\frac{\sqrt{\Lambda}}{4 \pi G m} \int d^{3} \boldsymbol{r}_{*}\left[\frac{1}{2} \mu_{*} \rho\right]=\frac{1}{2} N m \mu_{*}=\frac{1}{2} N \mu
$$

The mass of the boson star is given in the present Newtonian scheme by

$$
M=N m+E
$$

After substituting the solution (3.12) into (3.14), we find that the mass of the spherical boson star becomes

$$
M(N)=m N-\frac{G m^{3}}{\sqrt{2} \pi \sqrt{\Lambda}} N^{2} .
$$

Incidentally, if we can vary the value of $N$, the maximum of $M$ occurs for

$$
N=\frac{\pi}{\sqrt{2}} \frac{\sqrt{\Lambda}}{G m^{2}} .
$$

The maximum value of $M$ is

$$
M_{\max }=\frac{\pi}{2 \sqrt{2}} \frac{\sqrt{\Lambda}}{G m},
$$


which is supposed to be a typical mass of the boson star in the large coupling limit. This value for the mass of the boson star is several times greater than the general relativistic result [17]. This is very similar to the known case for the Newtonian and relativistic boson stars with no self-interaction [4]. We need not consider the boson star with the maximum mass, especially for explaining the galaxy rotation caused by a single huge boson star located at the center of the galaxy [9-11].

In $D$-dimensional spacetime, we take the same forms of the Lagrangian density $\mathcal{L}$ and the Hamiltonian density $\mathcal{H}$ as the four-dimensional ones. Then, the shape of the equation of motion for $\rho$ is unchanged; the rescaled Laplacian in the spherical system of $(D-1)$ dimensional space is replaced by

$$
\nabla_{*}^{2}=\frac{1}{r_{*}^{D-2}} \frac{d}{d r_{*}} r_{*}^{D-2} \frac{d}{d r_{*}} .
$$

For a $D$-dimensional boson star, the particle number $N$ is expressed as

$$
N=\frac{(\sqrt{\Lambda})^{D-3}}{4 \pi G m^{D-2}} \int d^{D-1} \boldsymbol{r}_{*} \rho\left(\boldsymbol{r}_{*}\right) .
$$

By solving the higher-dimensional equation, we find the solution

$$
\rho\left(r_{*}\right)=\left\{\begin{array}{cc}
\frac{4 \pi G m^{D-2}}{A_{D-1}(\sqrt{\Lambda})^{D-3}} \frac{2^{\frac{D-1}{2}} N}{q^{\frac{D-1}{2}} J_{\frac{D-1}{2}}(q)} \frac{J_{\frac{D-3}{2}}\left(\sqrt{2} r_{*}\right)}{\left(\sqrt{2} r_{*}\right)^{\frac{D-3}{2}}} & \left(r_{*}<\frac{q}{\sqrt{2}}\right) \\
0 & \left(r_{*}>\frac{q}{\sqrt{2}}\right)
\end{array},\right.
$$

and

$$
\Phi\left(r_{*}\right)=\left\{\begin{array}{cc}
-\frac{4 \pi G m^{D-2} N}{A_{D-1}(\sqrt{\Lambda})^{D-3}} \frac{2^{\frac{D-3}{2}}}{(D-3) q^{D-3}}-\frac{2 \pi G m^{D-2}}{A_{D-1}(\sqrt{\Lambda})^{D-3}} \frac{2^{\frac{D-1}{2}} N}{q^{\frac{D-1}{2}} J_{\frac{D-1}{2}}(q)} \frac{J_{\frac{D-3}{2}}\left(\sqrt{2} r_{*}\right)}{\left(\sqrt{2} r_{*}\right)^{\frac{D-3}{2}}} & \left(r_{*}<\frac{q}{\sqrt{2}}\right) \\
-\frac{4 \pi G m^{D-2} N}{A_{D-1}(\sqrt{\Lambda})^{D-3}} \frac{1}{(D-3) r_{*}^{D-3}} & \left(r_{*}>\frac{q}{\sqrt{2}}\right)
\end{array},\right.
$$

where $A_{D-1} \equiv \frac{2 \pi \frac{D-1}{2}}{\Gamma\left(\frac{D-1}{2}\right)}, J_{n}(z)$ is the Bessel function of the first kind, and $q$ is the first non-trivial zero of $J_{\frac{D-3}{2}}(x)$, i.e., $J_{\frac{D-3}{2}}(q)=0$. As in the case of $D=4$, we have chosen the value of $\mu_{*}$ so that $\Phi\left(r_{*}\right)$ matches the gravitational potential in vacuum outside the star.

The Newtonian energy is the same as that in four dimensions:

$$
E=\frac{1}{2} N m \mu_{*}=\frac{1}{2} N \mu \text {. }
$$

Thus, the mass of the spherical boson star is given by

$$
M(N)=N m-\frac{4 \pi G m^{D-1}}{2 A_{D-1}(\sqrt{\Lambda})^{D-3}} \frac{2^{\frac{D-3}{2}}}{(D-3) q^{D-3}} N^{2} .
$$


The maximum of $M$ occurs when

$$
N=\frac{A_{D-1}(\sqrt{\Lambda})^{D-3}}{4 \pi G m^{D-2}} \frac{(D-3) q^{D-3}}{2^{\frac{D-3}{2}}},
$$

and the maximum mass is

$$
M_{\max }=\frac{A_{D-1}(\sqrt{\Lambda})^{D-3}}{4 \pi G m^{D-3}} \frac{(D-3) q^{D-3}}{2^{\frac{D-1}{2}}} .
$$

\section{ROTATING NEWTONIAN BOSON STARS WITH LARGE SELF-INTERACTION}

Now we turn to the case of $D=4$ again and consider rotating boson stars. We assume their axial symmetry and equatorial symmetry.

For a stationary rotating boson star, we set [12-16]

$$
\Psi \rightarrow \Psi\left(r_{*}, \theta\right) e^{i s \varphi}
$$

where $\varphi$ indicates the polar angle and an integer $s$ corresponds to the angular momentum. Substituting this ansatz, Eq. (3.4) reads

$$
-\frac{1}{2 \Lambda}\left(\frac{1}{r_{*}} \frac{\partial^{2}}{\partial r_{*}^{2}} r_{*} \Psi+\frac{1}{r_{*}^{2} \sin \theta} \frac{\partial}{\partial \theta} \sin \theta \frac{\partial}{\partial \theta} \Psi-\frac{s^{2}}{r_{*}^{2} \sin ^{2} \theta} \Psi\right)+\Phi \Psi+\frac{1}{2}|\Psi|^{2} \Psi=\mu_{*} \Psi .
$$

We assume the large coupling limit $\Lambda \rightarrow \infty$ as well as the rapid rotation of the phase of the scalar field such that $s_{*}^{2} \equiv s^{2} / \Lambda$ takes a finite value. Then, the equation becomes

$$
\left[\Phi+\frac{1}{2}\left(|\Psi|^{2}+\frac{s_{*}^{2}}{r_{*}^{2} \sin ^{2} \theta}\right)-\mu_{*}\right] \Psi=0 .
$$

If the relation for $\Psi \neq 0$ is substituted into the Poisson equation, we obtain the following inhomogeneous differential equation:

$$
\nabla_{*}^{2} f+2 f=\frac{2 s_{*}^{2}}{r_{*}^{2} \sin ^{2} \theta}
$$

where

$$
f\left(r_{*}, \theta\right) \equiv \rho+\frac{s_{*}^{2}}{r_{*}^{2} \sin ^{2} \theta},
$$

with $\rho \equiv|\Psi|^{2}$.

The particular solution of (4.4) is given by [18]

$$
s_{*}^{2} f_{p}(x) \equiv-\frac{s_{*}^{2} \pi}{2} Y_{0}(\sqrt{2} x) G_{13}^{20}\left(\begin{array}{c|c}
x^{2} & 1 \\
2 & 0,0,0
\end{array}\right),
$$


where $x \equiv r_{*} \sin \theta, Y_{n}(z)$ is the Bessel function of the second kind and $G_{p q}^{m n}$ is the Meijer $G$ function.

The general solution to Eq. (4.4) with axial and equatorial symmetries $\left(\rho\left(r_{*}, \theta\right)=\rho\left(r_{*}, \pi-\right.\right.$ $\theta))$ is expressed by the particular solution $s_{*}^{2} f_{p}(x)$ plus the linear combination

$$
\sum_{n=0}^{\infty} j_{2 n}\left(\sqrt{2} r_{*}\right) P_{2 n}(\cos \theta)
$$

where $j_{l}(z)$ is the spherical Bessel function ${ }^{3}$ and $P_{n}(x)$ is the Legendre polynomial of the $n$-th order, or

$$
\int d k\left[\alpha(k) J_{0}\left(\sqrt{2-k^{2}} x\right)+\beta(k) Y_{0}\left(\sqrt{2-k^{2}} x\right)\right] \cos k z,
$$

where $z \equiv r_{*} \cos \theta$.

To simplify our analysis, we wish to use the minimal number of parameters throughout the present paper. The radius of the boson star is thought to be an important physical parameter. Hence, we first consider a simple ansatz that $\rho$ is given by

$$
A \frac{\sin \sqrt{2}\left(r_{*}-h\right)}{\sqrt{2} r_{*}}+s_{*}^{2}\left(f_{p}(x)-\frac{1}{x^{2}}\right)-s_{*}^{2}\left(f_{p}\left(R_{*}\right)-\frac{1}{R_{*}^{2}}\right) \frac{Y_{0}(\sqrt{2} x)}{Y_{0}\left(\sqrt{2} R_{*}\right)},
$$

where the scale factor $A$ is a dimensionless constant if its numerical value is positive; otherwise, $\rho=0$. Here, the equatorial radius of the boson star is given by $R_{*}=\frac{\pi}{\sqrt{2}}+h$.

As a bolder approximation, we will omit $f_{p}$ in the previous ansatz. The special solution $f_{p}(x)$ behaves logarithmic in the vicinity of the origin. The term proportional to $1 / x^{2}$ makes $\rho$ vanish more rapidly near the origin (Fig. 1 ). We can therefore omit $f_{p}(x)$ to make

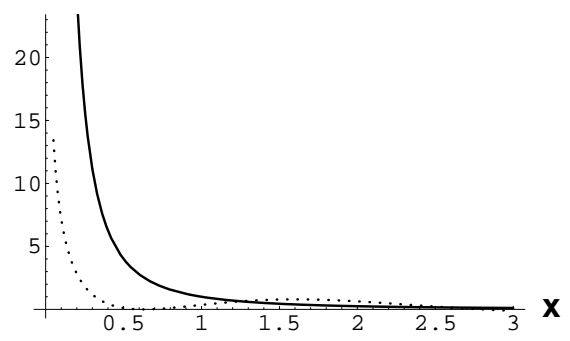

FIG. 1. $f_{p}(x)$ (the dotted line) and $1 / x^{2}$ (the solid line).

numerical integration efficient, and we finally adopt the following approximation for $\rho$ :

$$
A \frac{\sin \sqrt{2}\left(r_{*}-h\right)}{\sqrt{2} r_{*}}-s_{*}^{2}\left(\frac{1}{x^{2}}-\frac{1}{R_{*}^{2}} \frac{Y_{0}(\sqrt{2} x)}{Y_{0}\left(\sqrt{2} R_{*}\right)}\right),
$$

${ }^{3}$ Note that $j_{0}(z)=\sqrt{\frac{\pi}{2 z}} J_{1 / 2}(z)=\frac{\sin z}{z}$. 
if its value is positive.

The total particle number is still given by (3.9), as in the non-rotating case, while the Newtonian energy of the rotating boson star becomes

$$
E=\frac{\sqrt{\Lambda}}{4 \pi G m} \int d^{3} \boldsymbol{r}_{*}\left[\frac{1}{2} \rho\left(\mu_{*}+\frac{s_{*}^{2}}{2 x^{2}}\right)\right],
$$

which is deduced from (2.10) and field equations.

Now, we solve the Poisson equation (3.8) with the $\rho$ found above in order to evaluate the value of $\mu_{*}$. The solution for the Poisson equation can be expressed as follows:

$$
\Phi\left(\boldsymbol{r}_{*}\right)=\int G\left(\boldsymbol{r}_{*}-\boldsymbol{r}_{*}{ }^{\prime}\right) \rho\left(\boldsymbol{r}_{*}^{\prime}\right) d^{3} \boldsymbol{r}_{*}{ }^{\prime},
$$

where the Green function $G$ satisfies

$$
\nabla_{*}^{2} G\left(\boldsymbol{r}_{*}-\boldsymbol{r}_{*}{ }^{\prime}\right)=\delta^{3}\left(\boldsymbol{r}_{*}-\boldsymbol{r}_{*}{ }^{\prime}\right)
$$

The Green function satisfying the condition $G \rightarrow 0$ at the spatial infinity is well known and is given by

$$
G\left(\boldsymbol{r}_{*}-\boldsymbol{r}_{*}{ }^{\prime}\right)=-\frac{1}{4 \pi\left|\boldsymbol{r}_{*}-\boldsymbol{r}_{*}^{\prime}\right|}
$$

or

$$
-\frac{1}{4 \pi\left|\boldsymbol{r}_{*}-\boldsymbol{r}_{*}{ }^{\prime}\right|}=-\sum_{l=0}^{\infty} \sum_{m=-l}^{l} \frac{1}{2 l+1} \frac{r_{<}^{l}}{r_{>}^{l+1}} Y_{l m}^{*}\left(\theta^{\prime}, \varphi^{\prime}\right) Y_{l m}(\theta, \varphi),
$$

where $r_{<}=\min \left(r_{*}, r_{*}{ }^{\prime}\right), r_{>}=\max \left(r_{*}, r_{*}{ }^{\prime}\right)$, and $Y_{l m}(\theta, \varphi)$ is the spherical harmonic function.

Because the density distribution has been assumed to be axially and equatorially symmetric, the gravitational potential $\Phi$ outside the boson star can be obtained as

$$
\Phi(x, z)=-\int_{0}^{\infty} \int_{0}^{\infty} \rho\left(x^{\prime}, z^{\prime}\right) \frac{2 K\left(-\frac{4 x x^{\prime}}{\left(x-x^{\prime}\right)^{2}+\left(z-z^{\prime}\right)^{2}}\right)}{\pi \sqrt{\left(x-x^{\prime}\right)^{2}+\left(z-z^{\prime}\right)^{2}}} x^{\prime} d x^{\prime} d z^{\prime}
$$

where $K(m)$ is the complete elliptic integral of the first kind. By numerically integrating (4.16), we can obtain the value of $\mu_{*}$ as

$$
\mu_{*}=\Phi\left(R_{*}, 0\right)
$$

Now, we can numerically calculate the Newtonian energy E. The shape of the boson star is determined by $h$ and $s_{*}^{2} / A$ in the form (4.10). ${ }^{4}$ We can evaluate the normalized Newtonian binding energy

$$
\frac{\sqrt{\Lambda}}{4 \pi G m^{3}} \frac{E}{N^{2}}
$$

${ }^{4}$ Note that the unit of $h$ is $\sqrt{\Lambda} / m$ (see Eq. (3.2)). 


\begin{tabular}{|c|c|c|c|c|c|c|}
\hline & \multicolumn{6}{|c|}{$s_{*}^{2} / A$} \\
\hline & 0.3 & 0.5 & 0.7 & 0.9 & 1.1 & 1.3 \\
\hline 0.00 & $-0.0158283-0.0116258$ & -0.00630139 & -0.000286516 & 0.00780719 & 0.0163551 & 0.0256774 \\
\hline 0.05 & $-0.0157169-0.0121735$ & -0.00791623 & -0.00358682 & 0.00244601 & 0.00825089 & 0.0142766 \\
\hline 0.10 & $-0.0155833-0.0126021$ & -0.0092137 & -0.00551656 & -0.00165121 & 0.00226248 & 0.00613015 \\
\hline 0.15 & $-0.0154328-0.0129336$ & -0.0102595 & -0.00751525 & -0.00480722 & -0.00221123 & 0.000229342 \\
\hline$h \mid 0.20$ & -0.0152694 -0.0131877 & -0.0111047 & -0.00910385 & -0.00724985 & -0.00557194 & -0.00407807 \\
\hline 0.25 & $-0.015097 \quad-0.0133802$ & -0.0117865 & -0.0103671 & -0.00913752 & -0.00809426 & -0.0072192 \\
\hline 0.30 & $-0.0149176-0.0135201$ & -0.0123334 & -0.0113593 & -0.0105769 & -0.00995592 & -0.00946259 \\
\hline 0.35 & $-0.0147352-0.0136173$ & -0.0127593 & -0.0121104 & -0.0116216 & -0.0112503 & -0.0109625 \\
\hline 0.40 & $-0.0145493-0.0136709$ & -0.0130539 & -0.0126215 & -0.0123162 & -0.0120917 & -0.0119222 \\
\hline
\end{tabular}

TABLE I. The values of $\left(\sqrt{\Lambda} /\left(4 \pi G m^{3}\right)\right) E / N^{2}$ for the four-dimensional boson star.

which is apparently independent of the scale factor $A$.

Table I lists the values of $\left(\sqrt{\Lambda} /\left(4 \pi G m^{3}\right)\right) E / N^{2}$ for the four-dimensional boson star as a function of $h$ and $s_{*}^{2} / A$. If $\left(\sqrt{\Lambda} /\left(4 \pi G m^{3}\right)\right) E / N^{2}$ is negative, the solution is considered to be energetically stable.

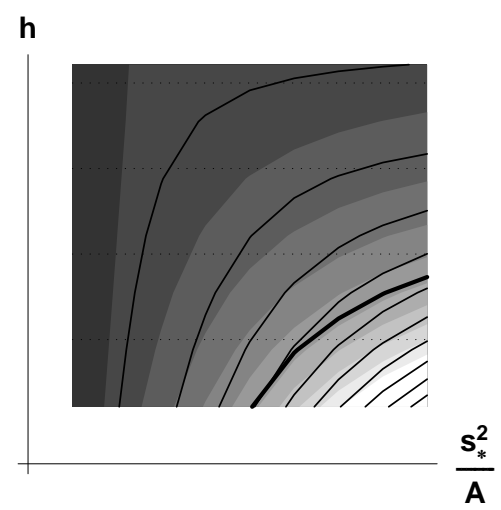

FIG. 2. The normalized Newtonian energy $E / N^{2}$ for the boson star in four dimensions shown by gray shades. On the thick line, the energy vanishes $(E=0)$. The dotted lines indicate contours of equal radii while the solid lines indicate contours of equal $s_{*}^{2} / N$. For the parameter region, please see the text.

Figure 2 shows this normalized Newtonian binding energy $\left(\sqrt{\Lambda} /\left(4 \pi G m^{3}\right)\right) E / N^{2}$ in a 
contour plot. The horizontal axis indicates $s_{*}^{2} / A=\{0.1,1.3\}$, while the vertical axis indicates $h=\{0.0,0.4\}$. A darker region corresponds to a lower energy. Each solid line shows the line on which the value $\frac{\sqrt{\Lambda}}{4 \pi G m^{2}} \frac{s_{*}^{2}}{N}=\frac{s^{2}}{4 \pi G m^{2} \sqrt{\Lambda} N}$ is constant.

For a small angular momentum or a small $s^{2} / N$, a lower $h$ yields a lower $E / N^{2}$. Thus, the equatorial radius of the boson star is $R_{*}=\pi / \sqrt{2}$ for a small $s^{2} / N$. For a large $s^{2} / N$, the binding energy $E$ becomes lower at a finite $h$. Under the condition that the conserved quantities, the particle number and angular momentum of the boson star, are fixed, the configuration with lower energy is considered to be realized. Therefore, for a fixed $N$, the equatorial radius of the rapidly rotating boson star increases with a higher angular momentum. This qualitative behavior corresponds to the result in the relativistic system studied by Ryan [15] in the large-coupling limit.

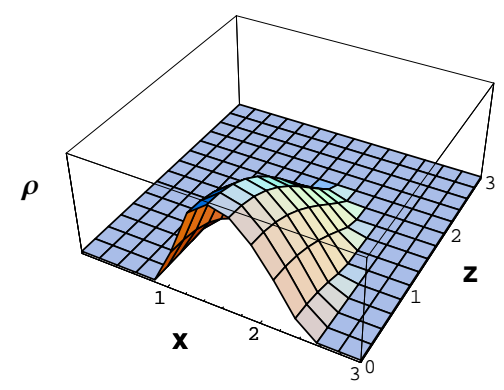

FIG. 3. A profile of the Newtonian rotating boson star with the parameters $h=0.25$ and $s_{*}^{2} / A=$ 0.8 .

We show a typical profile of the rotating boson star in Fig. 3, where the scale of the vertical axis is taken arbitrarily since the shape is independent of the scale factor $A$.

Next, we list the values of $4 \pi G \sqrt{\Lambda} m|Q| / s^{2}$, the ratio of the quadrupole moment to $s^{2}$ of the boson star, in Table II. The quadrupole moment $Q$ is given by

$$
Q=\frac{\sqrt{\Lambda}}{4 \pi G m} \int d^{3} \boldsymbol{r}_{*} \rho\left(\boldsymbol{r}_{*}\right)\left(3 z^{2}-\left|\boldsymbol{r}_{*}\right|^{2}\right)
$$

The ratio of the quadrupole moment to $s^{2}$ of the boson star is shown in Fig. 4 as a contour plot.

The parameter region is the same as in Fig. 2. From this plot, we find the following. For a small $s^{2} / N$, the ratio is small; when the radius of the boson star increases, the ratio increases. This qualitative behavior coincides with the relativistic result obtained by Ryan [15] in the large-coupling limit. Because the quadrupole moment is sensitive to the detail of 


\begin{tabular}{|l|c|ccccccc|}
\hline & \multicolumn{7}{|c|}{$s_{*}^{2} / A$} \\
\hline & 0.00 & 61.5431 & 26.9759 & 15.9985 & 10.7511 & 7.79774 & 5.96767 & 4.75667 \\
0.05 & 67.3542 & 30.3416 & 18.5505 & 12.867 & 9.63173 & 7.60095 & 6.23663 \\
0.10 & 73.5307 & 34.0246 & 21.426 & 15.3135 & 11.8044 & 9.5755 & 8.05857 \\
0.15 & 80.0819 & 38.0562 & 24.6637 & 18.1383 & 14.3593 & 11.9336 & 10.2657 \\
0.20 & 87.0118 & 42.4584 & 28.2936 & 21.3601 & 17.3033 & 14.667 & 12.8201 \\
0.25 & 94.3121 & 47.208 & 32.4701 & 24.9027 & 20.5056 & 17.5532 & 15.3778 \\
0.30 & 101.936 & 52.2142 & 36.3944 & 28.3421 & 23.2303 & 19.4039 & 16.4532 \\
0.35 & 109.702 & 56.997 & 39.5987 & 29.6371 & 22.7876 & 18.0043 & 14.5134 \\
0.40 & 117.032 & 59.4375 & 37.0806 & 24.1276 & 16.0474 & 10.5961 & 6.67022 \\
\hline
\end{tabular}

TABLE II. The value of $4 \pi G \sqrt{\Lambda} m|Q| / s^{2}$ for the four-dimensional boson star.

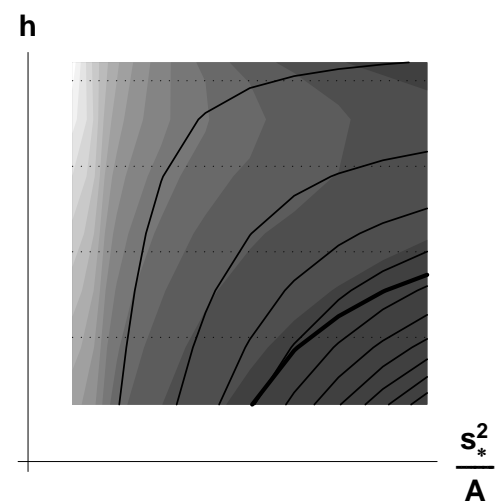

FIG. 4. The ratio of the quadrupole moment to $s^{2}, 4 \pi G \sqrt{\Lambda} m|Q| / s^{2}$ (see (4.19)), of the boson star shown by gray shades.

the shape, it is difficult to study on the qualitative correspondence to the previous result in the present analysis with the minimal number of parameters. 


\section{INTERLUDE: ON BOSON STARS IN THE KALUZA-KLEIN BACKGROUND}

In this section, we consider a boson-star solution in the Kaluza-Klein background, i.e., a boson star in spacetime with a compact extra dimension.

We denote the coordinate of the extra space as $y$. This dimension is assumed to be a circle, the circumference of which is set as $L$. Thus, the coordinate can be considered periodic by identifying $y+L \sim y$.

As in previous sections, we rescale the length scale as

$$
y_{*}=\frac{m}{\sqrt{\Lambda}} y, \quad L_{*}=\frac{m}{\sqrt{\Lambda}} L .
$$

The Lagrangian density is assumed to be of the same form as in the previous case. Accordingly, a non-zero $\rho$ satisfies the differential equation

$$
\left(\frac{1}{r_{*}^{2}} \frac{\partial}{\partial r_{*}} r_{*}^{2} \frac{\partial}{\partial r_{*}}+\frac{\partial^{2}}{\partial y_{*}^{2}}+2\right) \rho\left(r_{*}, y_{*}\right)=0
$$

if the spherical symmetry in three-dimensional space is assured. For a sufficiently small $L_{*}$, a solution for a boson star can be described by

$$
\rho=\max \left[A \frac{\sin \sqrt{2} r_{*}}{\sqrt{2} r_{*}}, 0\right]
$$

where the scale factor $A$ is a positive constant. This solution is the same as the fourdimensional solution; this configuration may appear like a 'boson string' in four-dimensional space.

On the other hand, for a finite length $L$, we conjecture the following solution for $\rho$ :

$$
\rho=\max \left[A\left(\frac{\sin \sqrt{2} r_{*}}{\sqrt{2} r_{*}}+\delta \frac{\sin \sqrt{2-\frac{4 \pi^{2}}{L_{*}^{2}}} r_{*}}{\sqrt{2} r_{*}} \cos \frac{2 \pi}{L_{*}} y_{*}\right), 0\right],
$$

if $\sqrt{2} \pi<L_{*}<2 \sqrt{2} \pi$. Here, $\delta$ is a constant. Note that at the special location $y_{*}=L_{*} / 4$, the surface of the boson star is located at $r_{*}=R_{*}=\pi / \sqrt{2}$.

Because of the periodicity in the extra coordinate $y_{*}$, the Green function for the Poisson equation defined in the Kaluza-Klein background is given by

$$
\begin{aligned}
G\left(\Delta \boldsymbol{r}_{*}, \Delta y_{*}\right) & =-\frac{1}{4 \pi^{2}} \sum_{n=-\infty}^{\infty} \frac{1}{\left|\Delta \boldsymbol{r}_{*}\right|^{2}+\left(\Delta y_{*}+n L_{*}\right)^{2}} \\
& =-\frac{\sinh \left(2 \pi\left|\Delta \boldsymbol{r}_{*}\right| / L_{*}\right)}{2 L_{*}^{2}\left(2 \pi\left|\Delta \boldsymbol{r}_{*}\right| / L_{*}\right)\left[\cosh \left(2 \pi\left|\Delta \boldsymbol{r}_{*}\right| / L_{*}\right)-\cos \left(2 \pi y_{*} / L_{*}\right)\right]},
\end{aligned}
$$


where $\left|\Delta \boldsymbol{r}_{*}\right|=\left|\boldsymbol{r}_{*}-\boldsymbol{r}_{*}^{\prime}\right|$ and $\Delta y_{*}=y_{*}-y_{*}^{\prime}$.

Using the assumption that $\rho$ depends on $r_{*}$ and $y_{*}$, and has the symmetry $y_{*} \leftrightarrow-y_{*}$, we obtain the gravitational potential, with an approximation of taking only the longest-wave mode, which is equivalent to neglecting the $O\left(\left(e^{-\left(2 \pi r_{*} / L_{*}\right)}\right)^{2}\right)$ terms, as

$$
\begin{aligned}
& \Phi\left(r_{*}, y_{*}\right)=\int_{0}^{L_{*}} d y_{*}^{\prime} \int d^{3} \boldsymbol{r}_{*}^{\prime} G\left(\Delta \boldsymbol{r}_{*}, \Delta y_{*}\right) \rho\left(r_{*}, y_{*}\right) \\
& \approx-\frac{1}{L_{*} r_{*}} \int_{0}^{L_{*}} d y_{*}^{\prime} \int_{0}^{\infty} r_{*}^{\prime 2} d r_{*}^{\prime} \rho\left(r_{*}^{\prime}, y_{*}^{\prime}\right) \\
& -2 \frac{e^{-\left(2 \pi r_{*} / L_{*}\right)}}{L_{*} r_{*}} \cos \frac{2 \pi y_{*}}{L_{*}} \int_{0}^{L_{*}} d y_{*}^{\prime} \int_{0}^{\infty} r_{*}^{\prime 2} d r_{*}^{\prime} \cos \frac{2 \pi y_{*}^{\prime}}{L_{*}} \cdot \frac{\sinh \left(2 \pi r_{*}^{\prime} / L_{*}\right)}{2 \pi r_{*}^{\prime} / L_{*}} \rho\left(r_{*}^{\prime}, y_{*}^{\prime}\right) .
\end{aligned}
$$

Thus, at the lowest order, we find

$$
\Phi\left(r_{*}, L_{*} / 4\right) \approx-\frac{G m^{3}}{\Lambda L_{*} r_{*}} N
$$

where

$$
N=\frac{\Lambda}{4 \pi G m^{3}} \int_{0}^{L_{*}} d y_{*} \int d^{3} \boldsymbol{r}_{*} \rho\left(\boldsymbol{r}_{*}, y_{*}\right) .
$$

Thus, the Newtonian energy of the boson star becomes

$$
E=\frac{1}{2} \mu_{*} N=\frac{1}{2} \Phi\left(R_{*}, L_{*} / 4\right) N \approx-\frac{G m^{3}}{\sqrt{2} \pi \Lambda L_{*}} N^{2},
$$

up to $O\left(e^{-4 \pi R_{*} / L_{*}}\right)$.

We conclude that the change in energy caused by the variation of the highest wavelength in the direction of the extra dimension is very small and is independent of the magnitude of its amplitude $\delta$, when only the longest-wave mode in the gravitational potential is taken into consideration.

Although further independent analysis is needed for clarifying the instability in this boson string, the instability in the Kaluza-Klein background qualitatively found here reminds us of the Gregory-Laflamme instability in black strings [19].

\section{NEWTONIAN BOSON STARS WITH LARGE SELF-COUPLING IN FIVE DIMENSIONS}

In this section, we consider a rotating boson star in five dimensions. We consider a system governed by the same form of the Lagrangian density as in the previous sections. 
The coordinates in the five-dimensional spacetime are assumed to be

$$
d s^{2}=-d t^{2}+d r^{2}+r^{2}\left(d \theta^{2}+\sin ^{2} \theta d \varphi_{1}^{2}+\cos ^{2} \theta d \varphi_{2}^{2}\right)
$$

where $0 \leq \theta \leq \pi / 2,0 \leq \varphi_{1}<2 \pi$ and $0 \leq \varphi_{2}<2 \pi$. After rescaling and redefining the coordinates, the line element becomes

$$
\begin{aligned}
d s^{2} & =-d t^{2}+\frac{\Lambda}{m^{2}}\left\{d r_{*}^{2}+r_{*}^{2}\left(d \theta^{2}+\sin ^{2} \theta d \varphi_{1}^{2}+\cos ^{2} \theta d \varphi_{2}^{2}\right)\right\} \\
& =-d t^{2}+\frac{\Lambda}{m^{2}}\left(d x^{2}+d y^{2}+x^{2} d \varphi_{1}^{2}+y^{2} d \varphi_{2}^{2}\right)
\end{aligned}
$$

where $x=r_{*} \sin \theta$ and $y=r_{*} \cos \theta$.

Here, we assume

$$
\Psi \rightarrow \Psi\left(r_{*}, \theta\right) e^{i s_{1} \varphi_{1}+i s_{2} \varphi_{2}}
$$

and define $s_{1 *} \equiv s_{1} / \sqrt{\Lambda}$ and $s_{2 *} \equiv s_{2} / \sqrt{\Lambda}$. As in Sec. IV, the large coupling limit yields the following relation in this case:

$$
\Phi+\frac{1}{2}\left(\rho+\frac{s_{1 *}^{2}}{x^{2}}+\frac{s_{2 *}^{2}}{y^{2}}\right)-\mu_{*}=0,
$$

for the region of $\rho=|\Psi|^{2}>0$. The differential equation in this case reads

$$
\nabla_{*}^{2} f+2 f=\frac{2 s_{1 *}^{2}}{r_{*}^{2} \sin ^{2} \theta}+\frac{2 s_{2 *}^{2}}{r_{*}^{2} \cos ^{2} \theta}
$$

where

$$
f\left(r_{*}, \theta\right) \equiv \rho+\frac{s_{1 *}^{2}}{r_{*}^{2} \sin ^{2} \theta}+\frac{s_{2 *}^{2}}{r_{*}^{2} \cos ^{2} \theta},
$$

in the region of $\rho>0$. For simplicity, we will consider only the simplest case with $s_{1}=s_{2}$ in this paper.

We conjecture that the boson star is spherical in the limit of no rotation. Since the Laplacian for the spherical body reads

$$
\nabla_{*}^{2}=\frac{1}{r_{*}^{3}} \frac{\partial}{\partial r_{*}} r_{*}^{3} \frac{\partial}{\partial r_{*}}
$$

the spherical solution of Eq. (6.5) with $s_{1 *}=s_{2 *}=0$ can be written by

$$
\frac{A}{\sqrt{2} r_{*}}\left\{J_{1}\left(\sqrt{2} r_{*}\right)-\frac{J_{1}\left(\sqrt{2}\left(\frac{q}{\sqrt{2}}+h\right)\right)}{Y_{1}\left(\sqrt{2}\left(\frac{q}{\sqrt{2}}+h\right)\right)} Y_{1}\left(\sqrt{2} r_{*}\right)\right\},
$$

where $A$ and $h$ are constants. $q \approx 3.83171$ is the first zero of $J_{1}(x)$. 
Because the Laplacian can also be expressed as

$$
\nabla_{*}^{2}=\frac{1}{x} \frac{\partial}{\partial x} x \frac{\partial}{\partial x}+\frac{1}{y} \frac{\partial}{\partial y} y \frac{\partial}{\partial y}+\frac{1}{x^{2}} \frac{\partial^{2}}{\partial \varphi_{1}^{2}}+\frac{1}{y^{2}} \frac{\partial^{2}}{\partial \varphi_{2}^{2}},
$$

the special solution of $(6.5)$ is determined to be $s_{1 *}^{2} f_{p}(x)+s_{2 *}^{2} f_{p}(y)$, where $f_{p}(x)$ is defined by (4.6). We neglect the contribution of $f_{p}$ to the solution, as in Sec. IV. In Sec. IV, we parametrize the equatorial radius, but in the present case, because there are 'holes' in the direction of the $x$ and $y$ axes, we abandon the tuning of the radius as an input parameter.

For simplicity, we consider the case with $s_{1 *}=s_{2 *} \equiv s_{*}$. Hence, the boson star has spherical symmetry in the limit of no rotation, and we take an approximated solution:

$$
\frac{A}{\sqrt{2} r_{*}}\left\{J_{1}\left(\sqrt{2} r_{*}\right)-\frac{J_{1}\left(\sqrt{2} R_{*}\right)}{Y_{1}\left(\sqrt{2} R_{*}\right)} Y_{1}\left(\sqrt{2} r_{*}\right)\right\}-s_{*}^{2}\left(\frac{1}{x^{2}}+\frac{1}{y^{2}}\right),
$$

where $R_{*} \equiv q / \sqrt{2}+h$, provided that the value of (6.10) takes a positive value. Note that $R_{*}$ is not the radius of the boson star.

The physical quantities of the boson star can be derived as in the four-dimensional case. The particle number of the boson star is expressed by

$$
N=\frac{\Lambda}{4 \pi G m^{3}} \int d^{4} \boldsymbol{r}_{*} \rho\left(\boldsymbol{r}_{*}\right)
$$

while the Newtonian binding energy is given by

$$
E=\frac{\Lambda}{4 \pi G m^{2}} \int d^{4} \boldsymbol{r}_{*}\left[\frac{1}{2} \rho\left(\mu_{*}+\frac{s_{1 *}^{2}}{2 x^{2}}+\frac{s_{2 *}^{2}}{2 y^{2}}\right)\right],
$$

for arbitrary values of $s_{1 *}$ and $s_{2 *}$.

The value of the gravitational potential at the boson star surface, $\mu_{*}$, should be obtained using the Green function in the flat four-dimensional space. The Green function, which asymptotically vanishes, is expressed as

$$
\begin{aligned}
G\left(\boldsymbol{r}_{*}, \boldsymbol{r}_{*}^{\prime}\right) & =-\frac{1}{4 \pi^{2}} \frac{1}{\left|\boldsymbol{r}_{*}-\boldsymbol{r}_{*}^{\prime}\right|^{2}} \\
& =-\frac{1}{4 \pi^{2}} \frac{1}{x^{2}+x^{\prime 2}-2 x x^{\prime} \cos \left(\varphi_{1}-\varphi_{1}^{\prime}\right)+y^{2}+y^{\prime 2}-2 y y^{\prime} \cos \left(\varphi_{2}-\varphi_{2}^{\prime}\right)} .
\end{aligned}
$$

Owing to the two axial symmetries of the boson star configuration, we wish to integrate the Green function over two polar coordinates. There is, however, no known compact expression for the integration, contrary to the case of the three spatial dimensions in Sec. IV. Thus, we take a further approximation. 
For $r_{*}^{\prime} \ll r_{*}$, the integration over the polar coordinates yields

$$
\begin{aligned}
& \frac{1}{(2 \pi)^{2}} \int_{0}^{2 \pi} d \varphi_{1}^{\prime} \int_{0}^{2 \pi} d \varphi_{2}^{\prime} G\left(\boldsymbol{r}_{*}, \boldsymbol{r}_{*}^{\prime}\right) \\
& =-\frac{1}{4 \pi^{2}} \frac{1}{r_{*}^{2}}-\frac{1}{4 \pi^{2}} \frac{2\left(x^{2} x^{\prime 2}+y^{2} y^{\prime 2}\right) / r_{*}^{2}-r_{*}^{\prime 2}}{r_{*}^{4}}+O\left({r_{*}^{\prime}}^{4} / r_{*}^{6}\right) .
\end{aligned}
$$

Hence, if $\theta=\tan ^{-1} x / y=\pi / 4$,

$$
\left.\frac{1}{(2 \pi)^{2}} \int_{0}^{2 \pi} d \varphi_{1}^{\prime} \int_{0}^{2 \pi} d \varphi_{2}^{\prime} G\left(\boldsymbol{r}_{*}, \boldsymbol{r}_{*}^{\prime}\right)\right|_{\theta=\pi / 4}=-\frac{1}{4 \pi^{2}} \frac{1}{r_{*}^{2}}+O\left(r_{*}^{\prime 4} / r_{*}^{6}\right) .
$$

Therefore, under the assumption that the higher multipole moments are relatively small, we take an approximation

$$
\mu_{*} \approx-\frac{1}{4 \pi^{2}} \frac{1}{\bar{R}_{*}^{2}} \int d^{4} \boldsymbol{r}_{*} \rho\left(\boldsymbol{r}_{*}\right)=-\frac{1}{4 \pi^{2}} \frac{1}{\bar{R}_{*}^{2}} \frac{4 \pi G m^{3} N}{\Lambda},
$$

where $\bar{R}_{*}$ denotes the distance between the origin and the boson star surface in the direction of $\theta=\pi / 4 .^{5}$

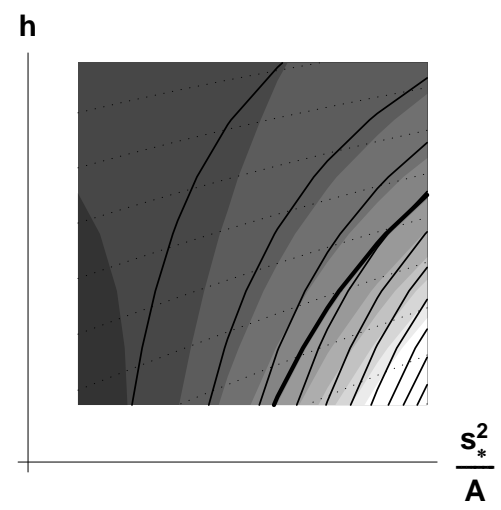

FIG. 5. The normalized Newtonian energy $E / N^{2}$ for the five-dimensional boson star shown by gray shades. On the thick line, the energy vanishes $(E=0)$. The dotted lines indicate contours of equal radii, while the solid lines indicate contours of equal $s_{*}^{2} / N$.

Table I lists the values of $\left(\Lambda /\left(4 \pi G m^{4}\right)\right) E / N^{2}$ for the five-dimensional boson star as a function of $h$ and $s_{*}^{2} / A$. If $\left(\Lambda /\left(4 \pi G m^{4}\right)\right) E / N^{2}$ is negative, the solution is considered to be energetically stable.

\footnotetext{
${ }^{5}$ For four-dimensional boson stars, the value of $\mu_{*}$ obtained through the surface potential at $\cos \theta=1 / \sqrt{3}$ agrees with the value obtained by integration within a deviation of at most ten percent in the region shown in Fig. 2.
} 


\begin{tabular}{|c|c|cccccccc|}
\hline & & \multicolumn{7}{|c|}{$s_{*}^{2} / A$} \\
\hline & & 0.01 & 0.02 & 0.03 & 0.04 & 0.05 & 0.06 & 0.07 & 0.08 \\
\hline & 0.1 & -0.00139555 & -0.00117091 & -0.000879967 & -0.00048938 & 0.0000448016 & 0.000796971 & 0.00185744 & 0.00342503 \\
& 0.2 & -0.00133381 & -0.00116085 & -0.000939441 & -0.000646538 & -0.000251982 & 0.000288229 & 0.00104247 & 0.00211638 \\
& 0.3 & -0.00127291 & -0.00114182 & -0.000976895 & -0.000763081 & -0.000481243 & -0.000105183 & 0.000407864 & 0.0011074 \\
& 0.4 & -0.0012138 & -0.001116 & -0.000996233 & -0.000844575 & -0.00064993 & -0.000398235 & -0.0000668219 & 0.000374118 \\
& 0.5 & -0.0011569 & -0.00108564 & -0.00100074 & -0.000896475 & -0.000766664 & -0.000603806 & -0.00039788 & -0.000134626 \\
& 0.6 & -0.0011025 & -0.00105179 & -0.000993517 & -0.000924326 & -0.00084106 & -0.000740175 & -0.000617337 & -0.000466712 \\
& 0.7 & -0.00105065 & -0.00101573 & -0.000977242 & -0.000933241 & -0.000882255 & -0.00082267 & -0.000752846 & -0.000670658 \\
\hline
\end{tabular}

TABLE III. The values of $\left(\Lambda /\left(4 \pi G m^{4}\right)\right) E / N^{2}$ for the five-dimensional boson star.

Figure 5 shows the normalized binding energy. The horizontal axis represents $s_{*}^{2} / A=$ $\{0.01,0.08\}$, while the vertical axis represents $h=\{0.1,0.7\}$. A darker region indicates a lower energy. Each contour shows the line on which the value $\frac{\Lambda}{4 \pi G m^{3}} \frac{s_{*}^{2}}{N}=\frac{s^{2}}{4 \pi G m^{3} N}$ is constant.

For a small $s^{2} / N$, a lower $h$ yields a lower $E / N^{2}$. Thus the radius $\bar{R}_{*}$ of the boson star is not significantly changed from the spherical case for a small $s^{2} / N$. For a large $s^{2} / N$, the Newtonian binding energy becomes lower at a finite $h$. This qualitative behavior is much similar to the case for the boson star in four dimensions.

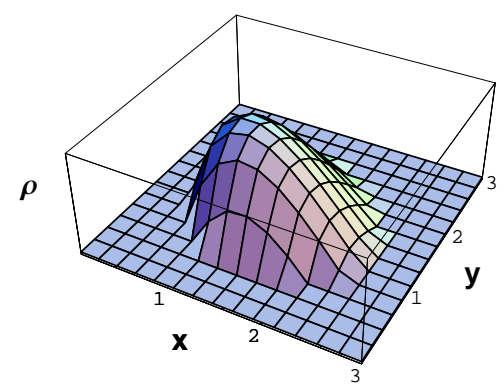

FIG. 6. A profile of the Newtonian rotating boson star with the parameters $h=0.4$ and $s_{*}^{2} / A=$ 0.06 .

Figure 6 shows a typical profile of the five-dimensional rotating boson star.

Brihaye and Hartmann reported [1] that the minimal boson star (in the case with no self-coupling of the scalar field) in five dimensions is energetically unstable. Although they attributed this instability to the power of the long-range tail of the gravitational potential, it is obvious that the stability depends on the balance between the self-interaction of the scalar field and the gravity. Therefore, the Newtonian approach in the higher dimensions will be a useful as well as significant method to study boson stars in models with general 
scalar potentials.

\section{SUMMARY AND PROSPECTS}

In this paper, Newtonian boson stars in the large coupling limit have been studied by means of various approximation methods. In summary, we found the qualitative behavior of the boson star parameters: 1) The Newtonian binding energy is determined by the particle number and the value of the gravitational potential, in addition to the energy from the rotation. 2) With a certain rapid rotation, the radius of the boson star becomes larger than that of the spherical boson star, if the particle number is fixed. 3) For a slowly rotating boson star, the radius is not significantly changed from that of the spherical one. 4) For a rotating four-dimensional boson star, the ratio of the quadrupole moment to the square of the angular momentum decreases when the angular momentum increases if its radius is unchanged from that of the spherical one. 5) The ratio of the quadrupole moment to the square of the angular momentum increases when the radius of the boson star increases.

We used Mathematica 4.2 [18] on a personal computer for numerical calculations. Of course, elaborate calculation on a large system would yield better results in terms of quality as well as quantity. In the present paper, since we used the minimal number of parameters to describe the deformation of the rotating boson star, we limit ourselves to a qualitative conclusion. The most important subject to be examined is the introduction of more parameters which determine the shape of the boson star in detail. This also depends on the computational environment.

The boson stars with finite self-coupling can also be approximated analytically by connecting the exponential tail in the asymptotic region of the boson star [20]. It is interesting to study the stability of the boson star in higher dimensions with analytical approximations in the Newtonian limit.

We wish to solve the configuration of the binary of the boson stars in a similar methods shown in this paper. The Newtonian treatment of the system would shed light on a possible relation among the physical quantities of the binary boson stars and provide an initial state for the dynamical calculation with general relativity or other theories of gravity. We also suppose that a rotating boson stars with a point mass at its center resembles a rotating black hole with scalar hair $[16,21,22]$. The study of such systems with the Newtonian 
approximation is interesting and may reveal an essential nature of gravity.

Finally, we anticipate that the Newtonian approach to the boson star would be valid for a multi-scalar system with many $U(1)$ charges, such as the 'multi-state boson star' [23], and the boson-fermion system [24-27], in order to extract its inherent characteristics in an efficient manner.

\section{ACKNOWLEDGMENTS}

We thank Prof. Carlos Herdeiro for valuable information on their work on rotating boson stars and hairy black holes [16, 21, 22].

[1] Y. Brihaye and B. Hartmann, Class. Quant. Grav. 33 (2016) 065002.

[2] B. Hartmann, J. Riedel and R. Suciu, Phys Lett. B726 (2013) 906.

[3] B. Hartmann, B. Kleihaus, J. Kunz and M. List, Phys. Rev. D82 (2010) 084022.

[4] P. Jetzer, Phys. Rep. 220 (1992) 163.

[5] A. R. Liddle and M. S. Madsen, Int. J. Mod. Phys. D1 (1992) 101.

[6] F. E. Schunck and E. W. Mielke, Class. Quant. Grav. 20 (2003) R301.

[7] F. E. Schunck and A. R. Liddle, Phys. Lett. B404 (1997) 25.

[8] F. E. Schunck and A. R. Liddle, "Boson stars in the centre of galaxies?" in "Black Holes: Theory and Observation", Proceedings of the Bad Honnef Workshop, F. W. Hehl, C. Kiefer and R. J. K. Metzler eds. (Springer-Verlag, Berlin, 1998), pp. 285-288, arXiv:0811.3764 [astro-ph].

[9] D. F. Torres, S. Capozziello and G. Lambiase, Phys. Rev. D62 (2000) 104012.

[10] F. E. Schunck and D. F. Torres, Int. J. Mod. Phys. D9 (2000) 601.

[11] T. Matos and L. A. Ureña-López, Gen. Rel. Grav. 39 (2007) 1279.

[12] F. E. Schunck, "Selbstgravitierende bosonische Materie" PhD-thesis, University of Cologne; 1996 (Cuvillier Press: Göttingen).

[13] F. E. Schunck and E. W. Mielke, "Rotating boson stars" in "Relativity and Scientific Computing: Computer Algebra, Numerics, Visualization", Proceedings of the Bad Honnef Workshop, F. W. Hehl, R. A. Puntigam and H. Ruder eds. (Springer-Verlag, Berlin, 1996), pp. 138-151.

[14] F. E. Schunck and E. W. Mielke, Phys. Lett. A249 (1998) 389. 
[15] F. D. Ryan, Phys. Rev. D55 (1997) 6081.

[16] C. A. R. Herdeiro, E. Radu and H. Rúnarsson, Phys. Rev. D92 (2015) 084059.

[17] M. Colpi, S. L. Shapiro and I. Wasserman, Phys. Rev. Lett. 57 (1986) 2485.

[18] Wolfram, http://www.wolfram.com/.

[19] R. Gregory and R. Laflamme, Phys. Rev. Lett. 70 (1993) 2837.

[20] F. S. Guzmán and L. A. Ureña-López, Phys. Rev. D68 (2003) 024023.

[21] C. A. R. Herdeiro and E. Radu, Phys. Rev. Lett. 112 (2014) 221101.

[22] C. A. R. Herdeiro and E. Radu, Class. Quant. Grav. 32 (2015) 144001.

[23] A. Bernal, J. Barranco, D. Alic and C. Palenzuela, Phys. Rev. D81 (2010) 044031.

[24] A. B. Henriques, A. R. Liddle and R. G. Moorhouse, Phys. Lett. B233 (1989) 99.

[25] A. B. Henriques, A. R. Liddle and R. G. Moorhouse, Nucl. Phys. B337 (1990) 737.

[26] A. B. Henriques, A. R. Liddle and R. G. Moorhouse, Phys. Lett. B251 (1989) 511.

[27] Ph. Jetzer, Phys. Lett. B243 (1990) 36. 